pendent of position) of radius $n^{1 / 2} / 2$ contains at least $N(n)$ lattice points on or within it?

II. Is it possible (for certain $n$ ) to replace the number $[n / 4]+1$ of the theorem by a number $M(n)$ which is greater than $[n / 4]+1$ ?

BROOKLYN, N. Y. AND

City College of the City of New York

\title{
ON THE MULTIPLICATIVE GROUP OF A DIVISION RING
}

W. R. SCOTT

Let $K$ be a noncommutative division ring with center $Z$ and multiplicative group $K^{*}$. Hua $[2 ; 3]$ proved that (i) $K^{*} / Z^{*}$ is a group without center, and (ii) $K^{*}$ is not solvable. A generalization (Theorem 1) will be given here which contains as a special case (Theorem 2) the fact that $K^{*} / Z^{*}$ has no Abelian normal subgroups. This latter theorem obviously contains both (i) and (ii). As a further corollary it is shown that if $M$ and $N$ are normal subgroups of $K^{*}$ not contained in $Z^{*}$, then $M \cap N$ is not contained in $Z^{*}$. The final theorem is that an element $x$ outside $Z$ contains as many conjugates as there are elements in $K$. This makes more precise a theorem of Herstein [1], who showed that $x$ has an infinite number of conjugates.

Square brackets will denote multiplicative commutation. If $S$ is a set, then $o(S)$ will mean the number of elements in $S$. A subgroup $H$ of $K^{*}$ is subinvariant in $K^{*}$ if there is a chain $\left\{N_{i}\right\}$ of subgroups such that $H \triangleleft N_{r-1} \triangleleft \cdots \triangleleft N_{1} \triangleleft K^{*}$, where $A \triangleleft B$ means that $A$ is a normal subgroup of $B$.

Lemma. Let $K$ be a division ring, $H$ a nilpotent subinvariant subgroup of $K^{*}, y \in H, x \in K^{*}$, and $[y, x]=\lambda \in Z^{*}, \lambda \neq 1$. Then the field $Z(x)$ is finite.

Proof. The proof of this lemma is essentially part of Hua's proof of (ii), but will be included for the sake of completeness.

Let $f$ be any rational function over $Z$ such that $f(x) \neq 0$. Then

Presented to the Society, November 23, 1956; received by the editors June 15, 1956. 


$$
\begin{aligned}
& x_{1}=[y, f(x)]=y f(x) y^{-1} f(x)^{-1}=f\left(y x y^{-1}\right) f(x)^{-1}=f(\lambda x) f(x)^{-1} ; \\
& x_{2}=\left[y, x_{1}\right]=f\left(\lambda^{2} x\right) f(\lambda x)^{-2} f(x) ;
\end{aligned}
$$

and, by induction, if $x_{n}=\left[y, x_{n-1}\right]$, then

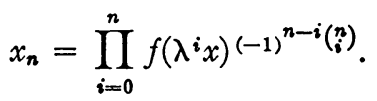

Now, by the subinvariance of $H, x_{1} \in N_{1}, x_{2} \in N_{2}, \cdots, x_{r} \in H$, and since $H$ is nilpotent, $x_{n}=1$ for some $n$. Letting $f(x)=1+x$, we have

$$
\Pi\left(1+\lambda^{i} x\right)^{\left(\begin{array}{l}
n \\
i
\end{array}\right)}-\Pi\left(1+\lambda^{i} x\right)^{\left(\begin{array}{l}
n \\
i
\end{array}\right)}=0,
$$

where the first product is taken over those $i$ such that $n-i$ is even and the second over those $i$ such that $n-i$ is odd, $i=0, \cdots, n$. In the left member, the constant term is equal to 0 , while the coefficient of $x$ is

$$
\sum_{i=0}^{n}(-1)^{n-i}\left(\begin{array}{l}
n \\
i
\end{array}\right) \lambda^{i}=(\lambda-1)^{n} \neq 0 .
$$

Thus $x$ is algebraic (of degree at most $2^{n-1}-1$ ) over $Z$. If $c \in Z^{*}$, then $[y, c x]=\lambda$, and $c x$ is also a root of (1). Therefore $Z$ is finite, and $Z(x)$ is finite as asserted.

THEOREM 1. Let $K$ be a division ring, $G$ and $H$ subinvariant subgroups of $K^{*}, x \in G, y \in H$, and $[y, x]=\lambda \in Z^{*} \lambda \neq 1$. Then one of $G$ and $H$ is not nilpotent.

Proof. Deny the theorem. By the lemma $Z$ is finite and both $x$ and $y$ are algebraic over $Z$. Since $y x=\lambda x y$, the set $S$ of elements of the form $\sum z_{i j} x^{i} y^{j}, z_{i j} \in Z$, is a finite noncommutative division ring.

THEOREM 2. If $K$ is a noncommutative division ring, then $K^{*} / Z^{*}$ has no normal Abelian subgroups.

Proof. If $N / Z^{*}$ is a normal Abelian subgroup, then $N$ is a nilpotent normal subgroup of $K^{*}$. The division ring generated by $N$ is invariant, hence by the Cartan-Brauer-Hua theorem is $K$ itself. Therefore $N$ is non-Abelian, and there are elements $x, y \in N$ such that $[y, x]=\lambda \in Z^{*}, \lambda \neq 1$. This contradicts Theorem 1 .

REMARK. The proof of Theorem 2 depends on Wedderburn's theorem that a finite division ring is a field. This can be avoided by the following considerations. Using the notation of the preceding proofs, $x_{1}=[y, 1+x] \in N, x_{2}=\left[y, x_{1}\right] \in Z, x_{3}=\left[y, x_{2}\right]=1$, and $n \leqq 3$. However, the coefficient of $x^{4}$ in the left member of (1) vanishes, so that $x$ and $y$ are of degree 2 over $Z$, and $o(Z)=3$ since it contains the distinct 
elements 0,1 , and $\lambda$. Then $o(S) \leqq 3^{4}$, and since $S$ must have room for a center and a subfield not in the center, $o(S)=3^{4}$ and $Z$ is the center of $S$. Thus $S^{*}$ is a group of order 80 and contains an element $u$ of order 5. The centralizer $C(u)$ of $u$ in $S$ is a division ring, hence of order 3,9 , or 81 , therefore by Lagrange's theorem of order 81 . But then $u \in Z, o(Z) \geqq 5$, and the contradiction proves the theorem.

Theorem 3. Let $K$ be a division ring and $M$ and $N$ be normal subgroups of $K^{*}$ not contained in $Z^{*}$. Then $M \cap N$ is not contained in $Z^{*}$.

Proof. Deny the theorem. Then $[M, N] \subset Z^{*}$. Let $y \in N, y \notin Z^{*}$. Since the centralizer $C(M)$ of $M$ is an invariant division ring not $K$, by the Cartan-Brauer-Hua theorem, $C(M)=Z$. Hence $y \notin C(M)$, and there is an $x$ in $M$ such that $[y, x]=\lambda \neq 1, \lambda \in Z^{*}$. The map $a \sigma=[x, a]$ is a homomorphism of $N$ into $Z^{*}$ with kernel $L \supset[N, N]$. Since $y \notin L, y \notin[N, N]$, and since $y$ was arbitrary, $[N, N] \subset Z^{*}$. Therefore $N$ is nilpotent. Similarly $M$ is nilpotent and Theorem 1 is contradicted.

Lemma. Let $K$ be an infinite division ring (perhaps commutative), $D$ a proper subdivision ring. Then $\left[K^{*}: D^{*}\right]=o(K)$.

Proof. Let $x \in K^{*}, x \in D^{*}$. Then the cosets $D^{*}(x+a), a \in D$, are distinct. Hence $\left[K^{*}: D^{*}\right] \geqq o(D)$. If $o(D)=o(K)$ we are done; if not, then $o(K)=o\left(K^{*}\right)=o\left(D^{*}\right)\left[K^{*}: D^{*}\right]$, hence again $\left[K^{*}: D^{*}\right]=o(K)$.

Theorem 4 (See [1]). If $K$ is a division ring and $x$ is a noncentral element, then $x$ has $o(K)$ conjugates.

Proof. The centralizer $C$ of $x$ is a proper subdivision ring of $K$. Then the number of conjugates of $x$ equals $\left[K^{*}: C^{*}\right]$ which is $o(K)$ by the lemma.

\section{BIBLIOGRAPHY}

1. I. N. Herstein, Conjugates in division rings, Proc. Amer. Math. Soc. vol. 7 (1956) pp. 1021-1022.

2. L. K. Hua, Some properties of a sfield, Proc. Nat. Acad. Sci. U.S.A. vol. 35 (1949) pp. 533-537.

3. - On the multiplicative group of a sfield, Science Record Acad. Sin. vol. 3 (1950) pp. 1-6.

UNIVERSITY OF KANSAS AND

INSTITUTE FOR ADVANCED STUDY 